\title{
Valoración y rediseño de una unidad sobre proporcionalidad, utilizando la herramienta Idoneidad Didáctica
}

\author{
Assessment and redesign of a unit on proportionality using the Didactical \\ Suitability tool
}

Avaliação e redesenho de uma unidade sobre proporcionalidade, usando a ferramenta de Idoneidade Didática

Daniel Esqué de los Ojos ${ }^{1}$ Adriana Breda ${ }^{2}$

Received: Dec/7/2019 • Accepted: Feb/8/2020 • Published: Jan/31/2021

\section{Resumen}

El objetivo de este trabajo es describir y analizar la reflexión que hace un profesor de su práctica, cuando utiliza los Criterios de Idoneidad Didáctica $(\mathrm{Cl})$ para valorar y rediseñar una unidad didáctica sobre la proporcionalidad. El análisis cualitativo del caso apunta que la valoración que hace el profesor de la unidad didáctica es bastante equilibrada, pues se pauta en todos los componentes de los $\mathrm{Cl}$. Sin embrago, al atribuir el peso al uso de los criterios en el rediseño de la unidad, el profesor pone más énfasis en la idoneidad epistémica, en particular, en el rediseño de diferentes tipologías de tareas con la finalidad de trabajar diferentes significados parciales de la proporcionalidad, en particular, el geométrico y el aritmético. Se concluye que este tipo de resultado puede ser observado en otras investigaciones que tratan de la reflexión que hace el profesor al utilizar la herramienta idoneidad didáctica.

Palabras clave: reflexión sobre la propia práctica; Criterios de Idoneidad Didáctica; formación de profesores de matemáticas; significados de la proporcionalidad.

\section{Abstract}

The objective of this paper is to describe and analyze the reflection made by a teacher while using the Didactical Suitability Criteria (DS) to assess and redesign a didactical unit of proportionality. The qualitative analysis points out that the assessment made by the teacher on the aforementioned unit is rather balanced, as it contemplates all the DS components. However, while weighting the different DS items used to redesign the unit, the teacher puts more emphasis on epistemic suitability, particularly, on redesigning different tasks typologies in order to explore a broader significance of proportionality, in particular, the geometric and arithmetic ones. It is concluded that this type of result can be observed

Daniel Esqué de los ojos, $\triangle$ daniel.esque@outlook.es, (1) https://orcid.org/0000-0002-6279-2037

Adriana Breda, $\triangle$ adriana.breda@ub.edu, (1) https://orcid.org/0000-0002-7764-0511

1 Institut Públic Vall d'Arús, Barcelona, España

2 Departamento de Educación Lingüística y Literaria, y Didáctica de las Ciencias Experimentales y de la Matemática, Universitat de Barcelona, Barcelona, España 
in other research projects dealing with the reflection that the teacher makes when using the didactical suitability tool.

Keywords: reflection on own practice; Didactical Suitability Criteria; training of mathematics teacher; meanings of proportionality.

\section{Resumo}

0 objetivo deste trabalho é descrever e analisar a reflexão que um professor faz de sua prática quando utiliza os Critérios de Idoneidade Didática (CI) para avaliar e redesenhar uma unidade didática sobre a proporcionalidade. A análise qualitativa do caso indica que a avaliação do professor sobre a unidade didática é bastante equilibrada, pois se baseia em todos os componentes do $\mathrm{Cl}$. No entanto, ao atribuir o peso ao uso dos critérios no redesenho da unidade, o professor enfatiza mais a adequação epistêmica, especialmente, 0 redesenho de diferentes tipologias de tarefas, a fim de trabalhar diferentes significados parciais da proporcionalidade, em particular, geométrico e aritmético. Conclui-se que esse tipo de resultado pode ser observado em outras pesquisas que tratam da reflexão que o professor faz ao utilizar a ferramenta de idoneidade didática.

Palavras-chave: reflexão sobre a própria prática; critérios de idoneidade didática; formação de professores de matemáticas; significados da proporcionalidade.

\section{INTRODUCCIÓN}

La investigación en el campo de la Educación Matemática está relacionada, sin duda, con el estudio sobre los conocimientos y las competencias didácticas y matemáticas que debe tener un profesor, para gestionar los procesos de enseñanza y aprendizaje. En ese sentido, se han generado en el área diversos modelos para caracterizar dichos conocimientos y competencias del profesor (Godino, Giacomone, Batanero \& Font, 2017; Rowland, Huckstep \& Thwaites, 2005; Hill, Ball \& Schilling, 2008; Schoenfeld \& Kilpatrick, 2008; Shulman, 1986). Entre los componentes propuestos por dichos modelos, uno de ellos se hace evidente: la reflexión del profesor sobre su práctica.

La reflexión del profesor sobre su propia práctica es competencia clave para el desarrollo profesional y la mejora de la enseñanza. Por esa razón, muchos enfoques teóricos se han centrado en investigar su desarrollo (Breda, Pino-Fan \& Font, 2017; Brockbank \& McGill, 2002; Davis, 2008; Elliott, 1993; Fortuny \& Rodríguez, 2012; Hart, Alston \& Murata, 2011; Llinares, 2012; Perrenoud, 2004; Schön, 1984).

Uno de los nuevos enfoques para el desarrollo de la competencia reflexiva del profesor de matemáticas es el Enfoque Ontosemiótico de la Cognición e Instrucción Matemática (EOS) (Godino, Batanero \& Font, 2007; 2019), lo cual nos proporciona los Criterios de Idoneidad Didáctica (CI) como una herramienta metodológica para pautar la reflexión sobre la práctica docente. Se tratan de criterios que pueden servir para guiar los procesos de enseñanza y aprendizaje de las matemáticas y para evaluar su implementación, tal como se viene haciendo en distintos procesos de formación en algunos países (Font, Breda \& Pino-Fan, 2017; Pochulu, Font \& Rodríguez, 2016; Seckel \& Font, 2015; 2020). 
Con el intento de verificar cómo se estructura la reflexión global sobre la práctica docente, el objetivo de este trabajo es describir y analizar la reflexión que hace un profesor de su propia práctica cuando utilizan los Criterios de Idoneidad Didáctica para valorar y rediseñar una unidad didáctica sobre la proporcionalidad.

En la próxima sección se registra una explicación de la herramienta Criterios de Idoneidad Didáctica (CI) y se hace una breve explicación sobre la complejidad del objeto matemático proporcionalidad. En la segunda, se muestran los aspectos metodológicos de este estudio, y en la tercera sección, se muestran los resultados y en la cuarta se hace una discusión sobre ellos proponiéndose algunas consideraciones finales.

\section{MARCO TEÓRICO}

\section{Criterios de Idoneidad Didáctica}

En el EOS se entiende la idoneidad didáctica de un proceso de enseñanza-aprendizaje como el grado en que este (o una parte de él) reúne ciertas características que permiten calificarlo como idóneo (óptimo o adecuado), para conseguir la adaptación entre los significados personales logrados por los estudiantes (aprendizaje) y los significados institucionales pretendidos o implementados (enseñanza), teniendo en cuenta las circunstancias y recursos disponibles (entorno). Los CI son considerados principios que pueden servir, primero, para guiar los procesos de enseñanza y aprendizaje de las matemáticas y, segundo, para valorar sus implementaciones (Breda, Font \& Pino-Fan, 2018).

En el EOS se consideran los siguientes CI (Font, Planas \& Godino, 2010): idoneidad epistémica, para valorar si las matemáticas que están siendo enseñadas son "buenas matemáticas". Idoneidad cognitiva, para valorar, antes de iniciar el proceso de instrucción, si lo que se quiere enseñar está a una distancia razonable de aquello que los alumnos saben, y, después del proceso, si los aprendizajes adquiridos están cerca de aquello que se pretendía enseñar. Idoneidad interaccional, para valorar si las interacciones resuelven dudas y dificultades de los alumnos. Idoneidad mediacional, para valorar la adecuación de los recursos materiales y temporales utilizados en el proceso de instrucción. Idoneidad emocional, para valorar la implicación (intereses y motivaciones) de los alumnos durante el proceso de instrucción. Idoneidad ecológica, para valorar la adecuación del proceso de instrucción al proyecto educativo del centro, las directrices curriculares, las condiciones del entorno social y profesional.

Para que estos criterios sean operativos en el ejercicio de análisis y valoración de los procesos de instrucción, se hace necesario su desglose en componentes e indicadores. Se detallan, en el Cuadro 1, los criterios y los componentes de idoneidad didáctica. El cuadro completo con los indicadores se puede encontrar en Breda y Lima (2016).

Tal como se explica en Breda et ál. (2018), las decisiones tomadas para delimitar las bases para el desarrollo del constructo CI fueron:

1) Una herramienta que permita al profesor reflexionar sobre su práctica y poder guiar su mejora en el contexto donde se realiza.

2) Utilizar un término que tenga un cierto aire de familia (Wittgenstein, 1953) con el de calidad, pero en el que los aspectos contextuales sean más predominantes que los estructurales o inherentes. Por 
Cuadro 1. Idoneidad Didáctica (CI): criterios y componentes

\begin{tabular}{lll}
\hline \multicolumn{1}{c}{ CI } & & Componente \\
\hline Epistémica & $\checkmark$ & Errores, ambigüedades, riqueza de procesos, representatividad \\
Cognitiva & $\checkmark$ Conocimientos previos, adaptación curricular a las diferencias individuales, aprendiza- \\
& $\quad$ je, alta demanda cognitiva \\
Interaccional & $\checkmark$ Interacción docente-discente, interacción entre discentes, autonomía, evaluación formativa \\
Mediacional & $\checkmark$ Recursos materiales, número de estudiantes, horario y condiciones del aula, tiempo \\
Afectiva & $\checkmark$ Intereses y necesidades, actitudes, emociones \\
Ecológica & $\checkmark$ Adaptación al currículo, conexiones intra- e interdisciplinares, utilidad sociolaboral, \\
& \multicolumn{2}{c}{ innovación didáctica } \\
\hline
\end{tabular}

Nota: basado en Hummes, Font y Breda (2019).

esta razón, se optó por el término idoneidad para introducir el constructo CI.

3) Considerar que la Didáctica de la Matemática (DM) puede generar principios provisionales (un tipo de normas llamadas aquí, criterios de idoneidad) consensuados por un sector importante de la comunidad interesada en la educación matemática, que pueden servir primero para guiar los procesos de enseñanza y aprendizaje de esta materia y, segundo, para valorar su implementación.

4) El constructo CI debe ser multidimensional y descomponerse en idoneidades parciales y, a su vez, cada una de ellas en componentes e indicadores (Godino, 2013).

5) Un proceso de enseñanza y aprendizaje se considera idóneo cuando se consigue un equilibrio entre los diferentes criterios parciales de idoneidad, y no cuando solo se dan algunos de ellos.

6) Los criterios de idoneidad parciales (en tanto que consensos a priori) pueden entrar en conflicto con el contexto en que trabaja el profesor, lo cual conlleva, primero, tratar los CI de manera conjunta (y no como criterios independientes como frecuentemente, se hace en el caso de la calidad) y, segundo, a cuestionar o relativizar la validez de un determinado criterio en un contexto específico, lo cual lleva a dar pesos relativos diferentes a cada criterio en función del contexto. Esta sexta decisión es posible porque los CI se consideran como normas que son principios en lugar de normas que son reglas. Los principios tienen un aspecto de peso o importancia que las reglas no tienen, de modo que los conflictos entre principios se resuelven por peso.

7) La posible contradicción entre la quinta y la sexta decisión se puede resolver mediante el rediseño del proceso de enseñanza y aprendizaje. En efecto, de acuerdo con la sexta decisión, el mayor peso dado a algunos principios en función del contexto inclina las decisiones en una dirección. Ahora bien, los principios con menor peso sobreviven intactos, aun cuando no prevalezcan, lo cual permite darles más peso en un rediseño del proceso de enseñanza y aprendizaje de cara a una implementación futura más equilibrada.

En la revisión de la literatura realizada en Breda, Font y Lima (2015), la noción de Idoneidad didáctica ha tenido un impacto relevante en la formación de profesores en diferentes países (Mallart, Font \& Malaspina, 2015; Seckel \& Font, 2015; 2020; Pochulu et ál., 2016). Tal impacto, se evidencia, por 
un lado, en el uso de los CI en diferentes investigaciones sobre formación de profesores de matemáticas, en las cuales se usa dicho constructo, pero no se hace en el marco de un dispositivo formativo pensado, expresamente, para enseñar la idoneidad didáctica como herramienta para organizar la reflexión del profesor sobre su propia práctica (Breda, 2020; Morales \& Font, 2017; Morales-López \& Araya-Román, 2020; Moreira, Gusmão \& Font, 2018).

Por otro lado, se encuentran investigaciones sobre dispositivos formativos en los cuales se observa el uso de los criterios de idoneidad didáctica, como contenido a explicar, para organizar la reflexión del profesor sobre su propia práctica, en grados (Seckel y Font, 2020) y postgrados (Font, et ál., 2017; Giacomone, Godino y Beltrán-Pellicer, 2018; Godino, Giacomone, Font y Pino-Fan, 2018).

\section{La complejidad del objeto matemá- tico proporcionalidad}

Tal como se ha explicado anteriormente, tanto los componentes como los indicadores de los CI se han confeccionado teniendo en cuenta las tendencias, los principios y los resultados de la investigación en el área de Didáctica de las Matemáticas. En particular, para la idoneidad epistémica se ha tenido en cuenta un principio fundamental del EOS que, con los matices propios de cada enfoque, es (o puede ser) asumido por otros enfoques teóricos del área. Nos referimos al principio que se puede formular de la siguiente manera: los objetos matemáticos emergen de las prácticas, lo cual conlleva su complejidad (Font, Godino \& Gallardo, 2013; Rondero \& Font, 2015). De este principio se deriva un componente (representatividad), cuyo objetivo es que se tenga en cuenta, dentro de lo posible, dicha complejidad en el diseño y el rediseño de las secuencias didácticas (Pino-Fan, Castro, Godino y Font, 2013).

El componente Representatividad de la complejidad de los objetos matemáticos (entendido como pluralidad de significados parciales), se refiere al grado de representatividad e interconexión de los significados institucionales implementados (o pretendidos) respecto de un significado de referencia (Giacomone, et al., 2018). Cada uno de estos significados permite resolver tipos de problemas diferentes, por lo cual, si se quiere enseñar una muestra representativa de significados parciales es necesario presentar una muestra variada de problemas (Font, Breda \& Seckel, 2017).

Se han realizado diferentes investigaciones para profundizar en la complejidad de diferentes objetos matemáticos: números naturales (Godino, Font, Wilhelmi \& Arrieche, 2009), medidas (Alpízar-Vargas \& Morales-López, 2019), media aritmética (Rondero \& Font, 2015), límite (Contreras, García \& Font, 2012), optimización (Balcaza, Contreras y Font, 2017), Teorema de Tales (Font, Breda \& Seckel, 2017), derivada y antiderivada, así como la comprensión que tienen los estudiantes de dicha complejidad (Pino-Fan, Godino \& Font, 2011; Pino-Fan, Castro, Godino \& Font, 2013; Pino-Fan, Font, Gordillo, Larios \& Breda, 2018; Pino-Fan, Godino \& Font, 2018), inecuación (Monje, Seckel \& Breda, 2018) y, no menos importante, proporcionalidad (Burgos, Beltrán-Pellicer, Giacomone \& Godino, 2018; Burgos, Castillo, Beltrán-Pellicer \& Godino, 2020).

Con base en los estudios de Burgos et ál. (2020), desde la perspectiva epistémica, la proporcionalidad puede ser considerada según cinco enfoques: 
Informal-cualitativo, centrado en la comparación multiplicativa de las cantidades que intervienen en los problemas y en la comparación perceptiva, por ejemplo, de la semejanza de formas geométricas.

Geométrico, centrado en las razones y las proporciones entre segmentos, escalas y semejanza de figuras. Las tareas relacionadas con escalas, ampliaciones y reducciones de figuras preservando la forma, permiten avanzar desde la aproximación intuitiva y cualitativa (preproporcional) a un significado cuantitativo esencialmente multiplicativo (proporcional).

Matemáticas griegas, dentro de estas el concepto de proporcionalidad quedaba representado para $a: b$ :: $c: d$ (a es para $b$ así como c es para d). Esta representación se encuentra descrita en el libro $\mathrm{V}$ de los Elementos de Euclides.

Aritmético, centrado en la noción de razón y proporción. Una razón establece una comparación multiplicativa entre un par ordenado de cantidades de magnitudes (homogéneas o heterogéneas), cada una de las cuales viene expresada mediante un número real y una unidad de medida. Una proporción es la igualdad de dos razones. El enfoque aritmético del estudio de la proporcionalidad predomina en la mayoría de las propuestas curriculares e investigaciones, distinguiéndose esencialmente dos categorías de problemas:

$\checkmark \quad$ Problemas de comparación. En un problema de este tipo, se dan cuatro valores, relacionados de manera multiplicativa dos a dos, formando „dos razones. El procedimiento de resolución es totalmente aritmético: la proporción es una relación de igualdad entre dos razones, $\mathrm{a} / \mathrm{b}=\mathrm{c} / \mathrm{d}$, donde $\mathrm{a}, \mathrm{b}, \mathrm{c}$ y d son números enteros cualesquiera, $\mathrm{b}$ y $\mathrm{d}$ son distintos de cero, y las razones $\mathrm{a} / \mathrm{b}$ y c/d son relaciones multiplicativas entre los números a, b y c, d, respectivamente.

$\checkmark$ Problemas de valor faltante. En estos la proporción es una relación de igualdad entre dos razones, en la que uno de los términos es un valor desconocido (valor faltante). Por ejemplo, $\mathrm{a} / \mathrm{b}=\mathrm{c} / \mathrm{x}$, donde a, $\mathrm{b}$ y c son números enteros conocidos y $x$ es el valor que se pretende determinar.

Algebraico-funcional, centrado en la noción de función lineal. Desde el enfoque funcional, se asume que "el razonamiento proporcional supone un tipo de razonamiento en un sistema de dos variables entre las que existe una relación funcional lineal que permite obtener conclusiones sobre una situación o fenómeno que puede ser caracterizado por una razón constante" (Karplus, Pulos \& Stage, 1983, p. 192, citado en Burgos et ál., 2020, p. 6-7). El modelo matemático que responde a esta situación es una función $y=k x$, donde $k$ es la razón constante unitaria o constante de proporcionalidad.

En cuanto la representatividad de tareas relacionadas a la proporcionalidad, estas se pueden traducir, según Cramer y Post (1993) en: a) problemas de valor faltante, consisten en, dadas dos magnitudes y conociendo tres valores, encontrar un valor desconocido; b) Problemas de comparación, consisten en determinar qué pareja de magnitudes tiene una razón de proporcionalidad mayor o si estas son iguales (un ejemplo es la concentración de fruta en un zumo); c) problemas cualitativos de predicción o comparación, tienen una estructura similar a los dos anteriores, pero se pueden resolver sin que sean necesarios cálculos numéricos. 


\section{METODOLOGÍA}

Esta investigación, de abordaje cualitativa, se enfoca en el estudio de un caso que analiza la reflexión que hace un profesor en ejercicio (estudiante de un máster de formación de profesores de matemáticas de secundaria) de su propia práctica, al utilizar los CI, para valorar y rediseñar una UD sobre proporcionalidad. La UD fue implementada con estudiantes del primer año de secundaria de una escuela pública de Barcelona. De acuerdo con Ponte (1994) y Yin (2001), el estudio de caso se caracteriza por un análisis muy particular, en lo cual el investigador no pretende cambiar la situación, pero sí comprenderla tal como se presenta. El motivo de elección de este caso, en particular, se atribuye al detalle de las justificaciones que hace el profesor en su reflexión, es decir, es un buen ejemplo del tipo de reflexión y valoración que se pretendía desarrollar en el máster.

La reflexión que hizo el profesor se incluyó en su trabajo de fin de máster, que es producto de un análisis de la secuencia didáctica implementada en la asignatura de prácticas profesionales. Es decir, en el Máster Profesional para Formación de Profesores de Matemáticas de Secundaria de Catalunya, hay una asignatura de Prácticum, dónde el profesor pone en juego los conocimientos adquiridos en el curso, al implementar en la educación básica una unidad didáctica (UD). Luego, en la asignatura Investigación sobre la propia práctica el profesor recibe herramientas para desarrollar y ordenar la reflexión (descripción, análisis, valoración y rediseño) de la unidad implementada. Finalmente, en la asignatura Trabajo Fin de Máster (TFM) se presenta, documentalmente, dicha reflexión. Una de las herramientas para organizar la reflexión del profesor es el constructo Idoneidad Didáctica, enseñada en las asignaturas de Investigación sobre la propia práctica y TFM.

\section{Enseñanza del constructo Criterios de Idoneidad en el máster}

En el máster, se toman los CI como un contenido a enseñar con el objetivo de que sean usados como pauta para organizar la propia práctica del profesor. En las asignaturas Innovación e investigación sobre la propia práctica y Trabajo fin de máster (TFM), en lugar de presentar los criterios de idoneidad como principios ya elaborados, se crean espacios para su generación como resultado de consensos en el grupo (Font, et ál., 2017), de acuerdo con la siguiente secuencia:

a) Análisis de casos (sin teoría): se propone a los alumnos la lectura y el análisis de episodios de clase para que hagan un análisis a partir de sus conocimientos previos, sin suministrarles ninguna pauta para ello.

b) Emergencia de diferentes tipos de análisis didáctico (descriptivo, explicativo $y$ valorativo): la puesta en común de los análisis realizada por los diferentes grupos, permite observar como el gran grupo contempla estos tres diferentes tipos de análisis didáctico, aunque cada grupo solo contemple alguno de ellos.

c) Tendencias en la enseñanza de las matemáticas: los episodios analizados se han seleccionado de manera que los asistentes apliquen de manera implícita alguna de las tendencias actuales sobre la enseñanza de las matemáticas (Breda, et ál., 2018). Seguidamente, se hace observar a los asistentes que han utilizado alguna de estas tendencias de manera implícita. 
d) Teoría (criterios de idoneidad): se explica que los criterios de idoneidad didáctica deben ser entendidos como principios emanados del discurso argumentativo de la comunidad educativa, cuando esta está orientada a conseguir un consenso sobre lo que se puede considerar mejor. También, se explica que para el desarrollo del constructo idoneidad didáctica, se han considerado las tendencias actuales sobre la enseñanza de las matemáticas, los principios del National Council of Teachers of Mathematics [NCTM] (2000) y los aportes de los diferentes enfoques teóricos del área de Didáctica de las Matemáticas (Godino, 2013; Breda, et ál., 2018).

e) Lectura y comentario de partes de algunos trabajos final de máster de cursos anteriores en los que los futuros profesores de cursos anteriores utilizaron los criterios de idoneidad didáctica para valorar la UD que implementaron.

f) En las asignaturas Prácticas y Trabajo final de máster los alumnos utilizarán los CI para valorar su propia práctica, en concreto la unidad que han diseñado e implementado. Tienen que hacer un rediseño y mejorar algunos de los aspectos que la valoración realizada indica que se deben y pueden mejorar.

\section{ANÁLISIS Y RESULTADOS}

El profesor presenta la valoración y el rediseño de una UD sobre proporcionalidad, para un grupo de alumnos del primer año de la Enseñanza Secundaria Obligatoria (12-13 años). A continuación, mostramos el uso que el profesor hace de los CI (en particular, sus componentes) para valorar la UD implementada y para justificar los cambios que hizo en su rediseño, con el objetivo de mejorarla.

\section{Idoneidad epistémica}

En ese apartado el profesor hace una autovaloración de la calidad matemática de UD y para eso, reflexiona sobre cada componente presente en la idoneidad epistémica. Con relación en los Errores, el profesor, en su reflexión, argumenta que no observa prácticas que se puedan considerar como erróneas desde el punto de vista matemático. Por contra, en el caso de las Ambigüedades, el profesor considera que detectó, al menos, un caso de ambigüedad a la hora de explicar cómo encontrar las coordenadas de un punto, pues en la explicación de cómo encontrarlas argumenta que:

(...) dado un punto en el primer cuadrante primero se baja para encontrar la coordenada x sobre el eje horizontal o de abscisas y, luego, partiendo de nuevo del punto, se va hacia la izquierda para ver la coordenada y sobre el eje vertical o de ordenadas. (Profesor).

Reflexiona que el inconveniente de hacerlo así es que algún estudiante podría pensar que, al ir "hacia abajo" y "hacia la izquierda" los valores de las coordenadas serían negativos. Para solucionar esta ambigüedad, el profesor comenta que lo mejor es explicar cómo encontrar las coordenadas del punto al partir del origen de coordenadas, de este modo arriba, abajo, derecha e izquierda proporcionarían, de forma natural, el signo correcto de las coordenadas.

Para el componente Riqueza de procesos el profesor considera que el conjunto de actividades propuestas en la UD abarca un amplio abanico de procesos matemáticos relevantes (resolución de problemas, argumentación, razonamiento y prueba, conexiones). Por ejemplo, el profesor explica que en la actividad ¡Atrapa al vampiro! los alumnos 
deben hacer un proceso de modelización para encontrar distancias sobre un mapa dada su escala, es decir, él considera que trabaja la proporción fuera del ámbito matemático.

Con relación en la Representatividad el profesor hace un estudio previo sobre los significados parciales de la noción de proporcionalidad (geométrico, matemáticas griegas, aritmético, algebraico - funcional) y describe que en la UD no se ha tratado la proporcionalidad geométrica, tampoco se ha trabajado el significado de las matemáticas griegas, lo cual se podría entender como una contextualización histórica. Reflexiona que quizás habría tenido sentido explorar este significado parcial, ya que alumnos estaban cursando la asignatura optativa de Mitología Griega y todos habían tratado el período histórico de la Grecia Clásica dentro de la asignatura de Sociales. Por otra parte, el profesor argumenta que la proporcionalidad aritmética ha sido ampliamente, trabajada en la unidad, así como la proporcionalidad algebraica-funcional. Además, en la valoración del profesor, se evidencia que la mayoría de los problemas propuestos por él, para trabajar la proporcionalidad, son de la tipología de problemas de valor faltante.

Como propuesta de mejora de la idoneidad epistémica el profesor argumenta que intentaría, en un primer momento, resolver el problema de las ambigüedades, luego, trabajaría una mayor representatividad en los tipos de tareas planteadas, al proponer una variedad más rica de problemas para cubrir el mayor número posible de significados parciales dentro del campo de la proporcionalidad.

\section{Idoneidad cognitiva}

El profesor, en su reflexión, asume que la UD implementada ha contemplado los conocimientos previos, en particular los relacionados a las operaciones entre enteros, las representaciones de fracciones, procedimientos de simplificación de fracciones y argumenta que los alumnos no presentaban problemas con las operaciones combinadas, la determinación de múltiples y representación de las fracciones a partir de representaciones gráficas. En cambio, el profesor comenta que los alumnos mostraron no tener bien asimiladas las operaciones entre fracciones, en especial las sumas y las restas.

En referencia al componente Adaptación curricular a las diferencias individuales el profesor describe que la adaptación curricular se llevó a cabo en dos sentidos: (i) en el de los procesos, para tener en cuenta dificultades lingüísticas y de aprendizaje y (ii) al aportar actividades de refuerzo para alumnos que presentaran dificultades a la hora de alcanzar los significados matemáticos, así como actividades de más alta demanda cognitiva para aquellos alumnos con altas capacidades.

Para verificar el Aprendizaje, el profesor considera que en la UD se llevaron a cabo evaluaciones en diferentes puntos del proceso de aprendizaje, una evaluación inicial para saber cuáles son los conocimientos previos de los alumnos y las diferentes evaluaciones parciales para dar seguimiento al proceso de aprendizaje (resolución de problemas, para generar discusión en torno a los significados matemáticos de la proporcionalidad, tareas que podrían generar la capacidad de trabajo individual, y al final, hizo una evaluación sumativa final, para medir el nivel de logro de ciertas competencias-objetivo establecidas al principio de la UD).

Con relación al componente Alta demanda cognitiva, el profesor considera que durante el desarrollo de la UD se han favorecido los procesos de generalización, cambio de representación (de tabla de 
valores a representación gráfica), conexiones intramatemáticas (la razón de proporcionalidad y la pendiente en una función lineal) y conjeturación.

Como propuesta de mejora de la idoneidad cognitiva el profesor argumenta que solo se ha presentado una rúbrica que se utilizó en la evaluación final y comenta que sería adecuado, en una reformulación de la UD, plantear otras rúbricas que se pudieran utilizar en secuencias intermedias del proceso de aprendizaje, para usarlas como herramientas de evaluación formadora, donde los alumnos tuviesen un feedback que les permitiera conocer cuáles son aquellos aspectos en los que les conviene mejorar.

\section{Idoneidad interaccional}

El profesor valora positivamente la interacción docente-discente, y explica que eso se debió a una pausada exposición de los conceptos con un tono de voz suficientemente claro y potente. Además, explica que, si se veía la clase muy callada, esto se entendía como la no comprensión de lo que se estaba contando y, por lo tanto, se buscaban otras estrategias para transmitir correctamente la información. Argumenta, también, que siempre se procuraba promover la participación de todo el grupo de la clase, sin llegar al extremo de violentar a algún alumno. Explica, además que la interacción entre discentes se daba en dos formas: a) al tener un compañero resolviendo un ejercicio en la pizarra, el resto discutía si estaba de acuerdo o no con la resolución; b) en la resolución de actividades en grupos heterogéneos.

Con relación a la Autonomía el profesor considera que, a parte de los momentos de estudio personal que implicaban la realización de las tareas encomendadas durante las horas lectivas, algunas actividades promovían la autonomía, ya que exigía que los alumnos utilizasen lo que habían visto durante las clases para responder a las situaciones-problema planteadas (no solo de forma grupal, pero también, de forma individual) y argumenta que el alumnado desarrollaba la autonomía cuándo organizaba su tiempo de trabajo y establecía las conexiones necesarias entre el cuerpo teórico explicado durante las clases y las preguntas y los problemas planteados.

El profesor reflexiona que se dieron procesos de evaluación formativa, en particular, durante la corrección de ejercicios en la pizarra y de actividades que los alumnos entregaban y comentaban que, para el caso de algunas actividades, al ser en grupo, la evaluación se podía dar in situ, al posibilitar la incidencia en el proceso de aprendizaje del alumno. A pesar de ello, concluye que esto se hizo de forma poco sistemática y podría ser uno de los aspectos a mejorar en el rediseño.

Para el profesor, una nueva propuesta de la UD podría pasar por una mejora en la evaluación formativa. Dado el conjunto de actividades propuestas en la UD, argumenta que sería conveniente sistematizar la recogida y el posterior análisis de las producciones del alumnado. El análisis debería venir dado por unos criterios de evaluación previamente compartidos con los estudiantes, de tal manera que ellos pudiesen entender que es lo que están haciendo, por qué y bajo qué criterios se evaluarán sus producciones.

\section{Idoneidad mediacional}

Con relación a los Recursos materiales el profesor argumenta que, durante la implementación de la UD, el uso de recursos no fue muy variado. Por un lado, se utilizó la pizarra convencional para introducir conceptos teóricos y resolver ejercicios, por otro lado, el uso del ordenador se limitó a la visualización del vídeo Troncho 
y Poncho (González, 2012), para introducir el concepto de proporcionalidad directa utilizando diversas representaciones. El tercer medio que se utilizó fue la calculadora, para trabajar las tareas de porcentaje:

En el momento del uso de la calculadora el profesor observó que algunos alumnos, al utilizar calculadoras cientificas, confundian la tecla correspondiente a la coma decimal con la correspondiente a la de los minutos de la notación sexagesimal. Ese hecho le llevo a reflexionar sobre la importancia de incluir alguna clase para enseñar algunas nociones básicas de uso de calculadores (profesor).

El profesor también presenta una valoración acerca del número de alumnos, condiciones del aula y horario, y argumenta que el número de alumnos es adecuado. Además, propone la idea de trabajar con dinámicas de aula (trabajo colaborativo, por ejemplo), donde fuera conveniente tener dos profesoras en el aula. Con relación al componente Tiempo el profesor describe que no tuvo tiempo para trabajar la proporcionalidad inversa y que buena parte del tiempo, en los dos grupos, se destinó a dejar claro el concepto de proporcionalidad directa y en sus diferentes representaciones.

Como propuesta de mejora de la idoneidad mediacional el profesor propone una mayor variabilidad en el uso de los recursos materiales, sobre todo, de los manipulativos. En particular, en su reflexión argumenta que sería interesante incluir los cubos multilink (o similar), para mostrar a los alumnos el significado de la razón de proporción. También, describe que el concepto de la proporcionalidad aplicado al aumento y la disminución de tamaño, se podría tratar en el ámbito de la fotografía.

\section{Idoneidad emocional}

En esta sección el profesor explora hasta qué punto la implementación de la UD ha tenido en cuenta los rasgos emocionales de los estudiantes, como se sentían durante las clases, desde un punto de vista de lo que puede ser interesante para ellos, y el estado anímico con el que frecuentaban las clases. Para el profesor, las actividades contemplaban los Intereses y necesidades de los alumnos, ya que algunas actividades, por ejemplo, las de contexto literario, se podrían considerar estimulantes, pues podrían ser aplicables al día a día del alumno. Sin embargo, comenta que muchas otras tareas, aunque fuesen estimulantes para el profesor, no lo fueron para los estudiantes.

Con relación al componente Actitudes, el profesor argumenta que el alumnado tenía una motivación práctica para realizar las actividades propuestas y esmerarse en hacerlas bien, ya que los criterios de evaluación establecidos por el Departamento de Matemáticas del centro escolar, decían que la recopilación de los trabajos enviados como tareas y de aquellas hechas en la propia aula conforman el $30 \%$ de la nota. También, justifica que algunas actividades fomentaban la corresponsabilidad

(...) algunas de las actividades se hacían en grupo $y$, por lo tanto, el trabajo de cada uno de los miembros del grupo repercutía en la nota global del grupo para esa misma actividad (profesor).

Además, también argumenta que se promovía la perseverancia en tanto que se iba controlando la producción de los alumnos in situ, haciendo comentarios que los fueran guiando hacia la buena solución sin darla directamente, después de una iteración y se procuraba promover la discusión 
pidiendo que los alumnos argumentaran sus respuestas.

Tanto durante la corrección de las tareas encomendadas como durante la discusión de dudas que algunas y algunos alumnos pudieran tener, siempre se promovió la participación del máximo número posible de alumnos, procurando que no fueran aquellas personas con más facilidad por la asignatura las que respondieran siempre a las preguntas formuladas tanto por el profesor como por otros alumnos (profesor).

Con relación al componente Emociones el profesor argumenta que durante las clases siempre se fue muy cuidadoso con aquellos alumnos que, a pesar de sus dificultades de aprendizaje, tenían el conocimiento suficiente para responder a las preguntas formuladas durante las clases. En caso de que la respuesta fuera errónea se procuraba establecer un diálogo con el alumno para intentar que este reconociera el error y fuera capaz de dar una respuesta adecuada.

Como propuesta de mejora, el profesor señala que es muy probable que las actividades que se seleccionaron no fueron del todo significativas para el alumnado y que una mejora en el campo emocional sería buscar unas actividades que interpelen mejor a los alumnos.

Esto se podría hacer en conjunción con una mejora de la idoneidad epistémica y cognitiva (profesor).

\section{Idoneidad ecológica}

En este criterio el profesor tiene presente el componente Adaptación al currículo, ya que él argumenta que la UD atendió a las exigencias propuestas por el
Departamento de Matemáticas del centro educativo, argumenta además que trabajó las Conexiones intradisciplinares, ya que se han establecido cambios de representación al trabajar la proporcionalidad, no solo como una razón entre dos magnitudes (representada en forma de fracción o porcentaje), sino también, al conectar el tema de proporcionalidad con lo de funciones lineales, este siendo uno de los significados parciales de la proporcionalidad directa. Argumenta también, que la UD fomentó el desarrollo de la competencia en el conocimiento y la interacción con el mundo físico, al trabajar la proporcionalidad entre magnitudes para el estudio y la descripción del entorno físico, es decir, tuvo en cuenta las Conexiones interdisciplinares.

Con relación al componente Utilidad sociolaboral el profesor explica que la UD implementada, a través de las actividades propuestas, una utilidad sociolaboral, ya que con las tareas implementadas los alumnos habrán aprendido que detrás de un hecho tan común como el acto de cocinar, están las proporciones, para poder representar distancias entre ciudades de diferentes países, se debe recurrir a la proporcionalidad y que, cuando amplían o reducen una fotografía, lo que están haciendo es aplicar proporciones.

El componente Innovación didáctica que el profesor, al reflexionar sobre la unidad implementada, asume tener llevado a cabo es la introducción de una evaluación formadora, ya que en el momento de la evaluación sumativa final, utilizó la rúbrica utilizada para evaluar las producciones de los alumnos. Las producciones eran evaluadas por los propios estudiantes y trianguladas con la evaluación del profesor por medio del uso de una rúbrica; así, se podría observar si había grandes discrepancias entre la nota otorgada por los alumnos y la del profesor. 
Otro hecho innovador asumido por el profesor fue incorporar el trabajo en grupo dentro de la asignatura, lo que no suele ser frecuente en la asignatura de matemáticas.

Como propuesta de mejora el profesor hace una reflexión en la cual asume que se podría mejorar la innovación en la evaluación y en la organización del aula. Por ejemplo, sugiere que en el caso de la evaluación se podrían pactar con los alumnos los criterios de evaluación de la rúbrica. En cuanto a la organización del aula, se podría escoger un criterio mejor para formar los grupos de trabajo, con la finalidad de que los grupos se quedasen más compensados, tanto en paridad de sexo como en diversidad intelectual.

\section{CONCLUSIONES}

El profesor hace una valoración bastante completa y equilibrada de la UD implementada -ya que utiliza en su reflexión todos los componentes de los CI- presentando sugerencias para su mejora. Propone, en particular: la elaboración de rúbricas para cada una de las actividades propuestas con la finalidad de potenciar la evaluación formativa (idoneidad cognitiva); la sistematización de la recogida de datos llevada a cabo durante la evaluación formativa para detectar carencias y poder alterar la programación didáctica (idoneidad interaccional); la inclusión de actividades de tipo manipulativo y TIC, siempre supeditadas a los recursos que puede ofrecer el instituto (idoneidad mediacional); la búsqueda de actividades más significativas para el alumnado, de manera que estas sean más estimulantes (idoneidad emocional); la promoción de un pacto con el alumnado para decidir los criterios de evaluación y ofrecer más retroalimentación durante la implementación de la UD (idoneidad ecológica).
Aunque el profesor exponga posibles cambios dentro y en cada uno de los CI, el criterio que tiene mayor peso en el rediseño de la UD es el epistémico. Para mejorar dicha idoneidad, el profesor propone una nueva colección de ejercicios con el objetivo de aumentar la representatividad de significados parciales y de tareas, en particular los significados parciales geométrico $\mathrm{y}$ aritmético.

Para el significado geométrico, argumenta que uno de los conceptos básicos para tener en cuenta es el Teorema de Tales, al proponer trabajar problemas relacionados a la semejanza de triángulos, para determinar alturas de objetos inalcanzables; la lectura de mapas para el cálculo de distancias; la ampliación y la reducción de fotografías para introducir conceptos de transformaciones en el plano y de óptica aplicables a la vida cotidiana de los estudiantes.

Para el significado aritmético, el profesor explica que lo más adecuado es trabajar con magnitudes medibles, por ejemplo, trabajar la proporción entre la distancia recorrida y el tiempo que tarda en recorrerla un vehículo viajando a velocidad constante. El profesor también argumenta que al trabajar la proporcionalidad los estudiantes presentan una aplicación incorrecta de la regla de tres, pues no tienen claro las condiciones bajo las cuales dos magnitudes son proporcionales. Por esta razón, para trabajar el significado aritmético de proporcionalidad, él propone ejercicios que contemplan los diferentes tipos de tareas: problemas de valor a faltante, problemas de comparación y problemas cualitativos de predicción o comparación.

El hecho de que el profesor presente una reflexión equilibrada acerca de la UD implementada, está directamente relacionado con las instrucciones que tuvo en el máster de formación de profesores de 
secundaria, en particular, en el estudio de la herramienta CI en las asignaturas Innovación e investigación sobre la propia práctica y Trabajo fin de máster, resultado que corrobora con el trabajo de Godino, Batanero, Rivas y Arteaga (2013). Sin embargo, la propuesta de mejora que hace se relaciona, sobre todo, a la idea de peso atribuida al uso de la herramienta CI, explicada en Breda, et ál. (2018).

El profesor, al poner en acción la UD en un contexto particular, entiende que, para mejorar la enseñanza y el aprendizaje de la proporcionalidad, es importante potenciar la idoneidad epistémica, proponiendo, en el rediseño, una serie de diferentes tipologías de tareas que incluyen los significados geométrico y aritmético. Este tipo de resultado se puede observar, también, en otras investigaciones inclinadas a estudiar la reflexión que hace el profesor cuando utiliza la herramienta idoneidad didáctica (Beltrán, Godino \& Giacomone, 2018; Font, et ál., 2017; Font, Breda, Seckel \& Pino-Fan, 2018; Giacomone, Godino \& Beltrán-Pellicer, 2018), en particular, en el contexto de la enseñanza de la proporcionalidad (Burgos, et ál., 2018; Godino, Giacomone, Font \& Pino-Fan, 2018).

\section{DECLARACIÓN DE LA CONTRIBUCIÓN DE LOS AUTORES}

El porcentaje total de contribución para la conceptualización, la preparación y la corrección de este artículo fue el siguiente: D. E. O. $50 \%$, A. B. $50 \%$.

\section{DECLARACIÓN DE DISPO- NIBILIDAD DE LOS DATOS}

Los datos que respaldan los resultados de este estudio serán puestos a disposición por el autor correspondiente D. E. O., previa solicitud razonable.

\section{AGRADECIMIENTOS}

Trabajo realizado en el marco de los proyectos de investigación: PGC2018098603-B-I00 (MCIU/AEI/FEDER, UE) y REDICE18-2000 (ICE-UB).

\section{REFERENCIAS}

Alpízar-Vargas, M. \& Morales-López, Y. (2019). Teaching the Topic of Money in Mathematics Classes in Primary School. Acta Scientiae, 21(5), 102-127. doi: https://doi.org/10.17648/ acta.scientiae. 5262

Balcaza, T.; Contreras, A. \& Font, V. (2017). Análisis de libros de texto sobre la optimización en el bachillerato, Bolema, 31(59), 1061-1081. doi: http://dx.doi. org/10.1590/1980-4415v31n59a11

Beltrán-Pellicer, P.; Godino, J. D. y Giacomone, B. (2018). Elaboración de indicadores específicos de idoneidad didáctica en probabilidad: aplicación para la reflexión sobre la práctica docente. Bolema, 32(61), 526-548. doi: http:// dx.doi.org/10.1590/1980-4415v32n61a11

Breda, A. \& Lima, V. M. R. (2016). Estudio de caso sobre el análisis didáctico realizado en un trabajo final de un máster para profesores de matemáticas en servicio. REDIMAT - Journal of Research in Mathematics Education, 5(1), 74-10. Doi: http://dx.doi.org/10.17583/ redimat.2016.1955

Breda, A. (2020). Características del análisis didáctico realizado por profesores para justificar la mejora en la enseñanza de las matemáticas. Bolema, 34(66), 69-88. https://doi. org/10.1590/1980-4415v34n66a04

Breda, A.; Font, V. \& Lima, V. (2015). A noção de idoneidade didática e seu uso na formação de professores de matemática. Jornal 
Internacional de Estudos em Educação Matemática, 8(1), 4-41. Doi: http://dx.doi. org/10.17921/2176-5634.2015v8n2p\%25p

Breda, A.; Font, V. \& Pino-Fan, L. (2018) Criterios valorativos y normativos en la didáctica de las matemáticas: el caso del constructo idoneidad didáctica. Bolema, 32(60), 255-278. Doi: http://dx.doi. org/10.1590/1980-4415v32n60a13

Breda, A.; Pino-Fan, L. R. \& Font, V. (2017). Meta Didactic-Mathematical Knowledge of Teachers: Criteria for The Reflection and Assessment on Teaching Practice. EURASIA Journal of Mathematics, Science and Technology Education, 13, 1893-1918. Doi: https://doi. org/10.12973/eurasia.2017.01207a

Brockbank, A. \& McGill, I. (2002). Aprendizaje reflexivo en la educación superior. Madrid: Morata.

Burgos, M., Castillo, M. J., Beltrán-Pellicer, P. \& Godino, J. D. (2020). Análisis didáctico de una lección sobre proporcionalidad en un libro de texto de primaria con herramientas del enfoque ontosemiótico, Bolema, 34(66), 40-68. https://doi.org/10.1590/1980-4415v34n66a03

Burgos, M.; Beltrán-Pellicer, P.; Giacomone, B. \& Godino, J. D. (2018). Conocimientos y competencia de futuros profesores de matemáticas en tareas de proporcionalidad. Educação e Pesquisa, 44, 1-22. Doi: http://dx.doi. org/10.1590/s1678-4634201844182013.

Contreras, A.; García, M. \& Font, V. (2012) Análisis de un proceso de estudio sobre la enseñanza del límite de una función. Bolema, 26(42B), 667-690. Doi: 10.1590/ S0103-636X2012000200013.

Cramer, K., \& Post, T. (1993). Proportional reasoning. The Mathematics Teacher, 86(5), 404-407.

Davis, B. (2008). Is 1 a prime number? Developing teacher knowledge through concept study. Mathematics Teaching in the Middle School, 14(2), 86-91. Recuperado de: https://eric. ed.gov/?id=EJ809139

Elliot, J. (1993). El cambio educativo desde la investigación-acción. Madrid: Morata.

Font, V.; Breda, A. \& Pino-Fan, L. (2017). Análisis didáctico en un trabajo de fin de máster de un futuro profesor. En J. M. Muñoz-Escolano, A. Arnal-Bailera, P. Beltrán-Pellicer, M. L. Callejo y J. Carrillo (Eds.), Investigación en Educación Matemática XXI (pp. 255-264). Zaragoza: SEIEM.
Font, V.; Breda, A. \& Seckel, M. J. (2017). Algunas implicaciones didácticas derivadas de la complejidad de los objetos matemáticos cuando estos se aplican a distintos contextos. Revista Brasileira de Ensino de Ciência e Tecnologia (RBECT), 10(2), 1-23. Doi: 10.3895/rbect.v10n2.5981

Font, V.; Breda, A.; Seckel, M. J. \& Pino-Fan, L. R. (2018). Análisis de las reflexiones y valoraciones de una futura profesora de matemáticas sobre la práctica docente. Revista de Ciencia y Tecnología, 34(2), 62-75. Recuperado de: https://revistas.ucr.ac.cr/index.php/ cienciaytecnologia/article/view/36628/37325

Font, V.; Godino, J. D. \& Gallardo, J. (2013). The emergence of objects from mathematical practices. Educational Studies in Mathematics, 82(1), 97-124. Doi: 10.1007/ s10649-012-9411-0.

Font, V.; Planas, N. \& Godino, J. D. (2010). Modelo para el análisis didáctico en educación matemática. Infancia y aprendizaje, 33(1), 89-105. https:// doi.org/10.1174/021037010790317243

Fortuny, J. M. \& Rodríguez, R. (2012). Aprender a mirar con sentido: facilitar la interpretación de las interacciones en el aula. Avances de investigación en Educación Matemática, (1), 23-37. Doi: 10.35763/aiem.v1i1.3

Giacomone, B.; Godino, J. D. \& Beltrán-Pellicer, P. (2018). Developing the prospective mathematics teachers' didactical suitability analysis competence. Educação e Pesquisa, 44, e172011. Doi: 10.1590/ s1678-4634201844172011.

Godino, J. D. (2013). Indicadores de la idoneidad didáctica de procesos de enseñanza y aprendizaje de las matemáticas. Cuadernos de investigación y formación en educación matemática, (11), 111-132. Recuperado de: https://revistas.ucr.ac.cr/index.php/cifem/ article/view/14720.

Godino, J. D.; Batanero, C. \& Font, V. (2007). The onto-semiotic approach to research in mathematics education. ZDM- The International Journal of Mathematics Education, 39(1), 127 - 135. Recuperado de: https://link.springer.com/article/10.1007/s11858-006-0004-1.

Godino, J. D.; Batanero, C. \& Font, V. (2019). The Onto-semiotic Approach: implications for the prescriptive character of didactics. For the Learning of Mathematics, 39(1), 37-42. Recuperado de: https://eric.ed.gov/?id=EJ1211459 
Godino, J. D.; Font, V.; Wilhelmi, M. R. \& Arrieche, M. (2009) ¿Alguien sabe qué es el número? Unión, 19, 34-46. Recuperado de: http://www.fisem.org/www/union/revistas/2009/19/Union 019 008.pdf.

Godino, J. D.; Giacomone, B.; Batanero, C. \& Font, V. (2017). Enfoque ontosemiótico de los conocimientos y competencias del profesor de matemáticas. Boletim de Educação Matemática, 31(57), 90-113. Doi: 10.1590/1980-4415v31n57a05.

Godino, J. D.; Giacomone, B.; Font, V., \& Pino-Fan, L. (2018). Conocimientos profesionales en el diseño y gestión de una clase sobre semejanza de triángulos. Análisis con herramientas del modelo CCDM. Avances de investigación en educación matemática, (13), 63 - 83. Doi: 10.35763/aiem.v0i13.224

Godino, J. D; Batanero, C.; Rivas, H. \& Arteaga, P. (2013). Componentes e indicadores de idoneidad de programas de formación de profesores en didáctica de las matemáticas. REVEMAT, 8(1), 46-74. Doi: 10.5007/1981-1322.2013v8n1p46.

González, A. (2012). Las aventuras de Troncho y Poncho: proporcionalidad. [vídeo en línea]. Recuperado de: https://www.youtube.com/ watch? $\mathrm{v}=9 \mathrm{QjVXWqS8Q} 4$

Hart, L. C.; Alston, A. S. \& Murata, A. (2011). Lesson study research and practice in mathematics education. Netherlands: Springer. https:// doi.org/10.1007/978-90-481-9941-9

Hill, H. C.; Ball, D. L. \& Schilling, S. G. (2008). Unpacking pedagogical content knowledge: Conceptualizing and measuring teachers' topic-specific knowledge of students. Journal for research in mathematics education, 39 (4) 372-400. Recuperado: https://www.jstor.org/ stable/40539304

Hummes, V. B.; Font, V. \& Breda, A. (2019). Combined Use of the Lesson Study and the Criteria of Didactical Suitability for the Development of the Reflection on the own Practice in the Training of Mathematics Teachers, Acta Scientiae, 21(1), 64-82. Doi: 10.17648/acta. scientiae.v21iss 1id4968.

Karplus, R.; Pulos, S. \& Stage, E. K. (1983). Early adolescents' proportional reasoning on 'rate'problems. Educational studies in Mathematics, 14(3), 219-233. Recuperado de: https:/link. springer.com/article/10.1007/BF00410539
Llinares, S. (2012). Construcción de conocimiento y desarrollo de una mirada profesional para la práctica de enseñar matemáticas en entornos en línea. Avances de investigación en educación matemática, (2), 53-70. Doi: https:// doi.org/10.35763/aiem.v1i2.18

Mallart, A.; Font, V. \& Malaspina, U. (2015). Reflexión sobre el significado de qué es un buen problema en la formación inicial de maestros. Perfiles educativos, 38(152), 14-30. https://doi.org/10.22201/ iisue.24486167e.2016.152.57585

Monje, Y.; Seckel, M. J. \& Breda, A. (2018). Tratamiento de la inecuación en el currículum y textos escolares chilenos, Bole$m a$, 32(61), 480-502. Doi: http://dx.doi. org/10.1590/1980-4415v32n61a09.

Morales-López, Y. \& Font, V. (2017). Análisis de la reflexión presente en las crónicas de estudiantes en formación inicial en educación matemática durante su periodo de práctica profesional. Acta Scientiae, 19(1), 122-137. Recuperado e: http://www.periodicos.ulbra. br/index.php/acta/article/view/2975.

Morales-López, Y., \& Araya-Román, D. (2020). Helping Preservice Teachers to Reflect. Acta Scientiae, 22(1), 88-111. http://doi. org/10.17648/acta.scientiae.5641

Moreira, C. B.; Gusmão, T. C. R. S. \& Font, V. (2018). Mathematical Tasks for the Development of Space Perception in Early Childhood Education: potentials and limits. Bolema: Boletim de Educação Matemática, 32(60), 231-254. Doi: http://dx.doi. org/10.1590/1980-4415v32n60a12.

National Council of Teachers of Mathematics (2000). Principles and standards for school mathematics. Reston, VA: Author.

Perrenoud, P. (2004). Desarrollar la práctica reflexiva en el oficio de enseñar: profesionalización y razón pedagógica. Barcelona: Graó.

Pino-Fan, L. R.; Font, V.; Gordillo, W.; Larios, V. \& Breda, A. (2018). Analysis of the meanings of the antiderivative used by students of the first engineering courses. International Journal of Science and Mathematics Education, 16(6), 1091-1113. Doi: 10.1007/s10763-017-9826-2

Pino-Fan, L.; Castro, W. F.; Godino, J. D. \& Font, V. (2013). Idoneidad epistémica del significado de la derivada en el currículo de bachillerato. Paradigma, 34(2), 123 - 150. Recuperado de: 
http://ve.scielo.org/scielo.php?script=sci arttext\&pid=S1011-22512013000200008.

Pino-Fan, L.; Godino, J. D. \& Font, V. (2011). Faceta epistémica del conocimiento didáctico-matemático sobre la derivada. Educação Matemática Pesquisa, 13(1), 141-178. Recuperado de: http://ken.pucsp.br/emp/ article/view/4423.

Pino-Fan, L.; Godino, J. D. \& Font, V. (2018). Assessing key epistemic features of didactic-mathematical knowledge of prospective teachers: the case of the derivative. Journal of Mathematics Teacher Education, 21(1), 63-94. https://doi.org/10.1007/s10857-016-9349-8

Pochulu, M.; Font, V. \& Rodríguez, M. (2016). Desarrollo de la competencia en análisis didáctico de formadores de futuros profesores de matemática a través del diseño de tareas. Revista Latinoamericana de Investigación en Matemática Educativa-RELIME, 19(1), 71-98. Doi: http://dx.doi.org/10.12802/ relime.13.1913

Ponte, J. P. (1994). O estudo de caso na investigação em educação matemática. Quadrante, 3(1), 3-18. Recuperado de: https://quadrante.apm. pt/index.php/quadrante/article/view/410.

Rondero, C. \& Font, V. (2015). Articulación de la complejidad matemática de la media aritmética. Enseñanza de las ciencias: revista de investigación y experiencias didácticas, 33(2), 29-49. Doi: https://doi.org/10.5565/ rev/ensciencias. 1386
Rowland, T.; Huckstep, P. \& Thwaites, A. (2005). Elementary teachers' mathematics subject knowledge: The knowledge quartet and the case of Naomi. Journal of Mathematics Teacher Education, 8(3), 255-281. Doi: https://doi.org/10.1007/s10857-005-0853-5

Schoenfeld, A. H. \& Kilpatrick, J. (2008). Toward a theory of proficiency in teaching mathematics. In The Handbook of Mathematics Teacher Education, 2 (pp. 321-354). Brill Sense. https://doi.org/10.1163/9789087905460_016

Schön, D. A. (1984). The reflective practitioner: How professionals think in action. Routledge.

Seckel, M. J. \& Font, V. (2020). Competencia reflexiva en formadores del profesorado en matemáticas. Magis, 12(25), 127-144. Doi: 10.11144/Javeriana.m12-25.crfp

Seckel. M. J., \& Font, V. (2015). Competencia de reflexión en la formación inicial de profesores de matemática en Chile. Práxis Educacional, 11(19), 55-75. Recuperado de: http://diposit. ub.edu/dspace/handle/2445/108902.

Shulman, L. S. (1986). Those who understand: Knowledge growth in teaching. Educational researcher, 15(2), 4-14. Doi: https://doi. org/10.3102/0013189X015002004

Wittgenstein, L. (1953). Investigaciones filosóficas. Barcelona: Crítica.

Yin, R. (2001). Estudo de caso: planejamento e métodos. Porto Alegre: Bookman.

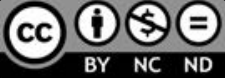

Valoración y rediseño de una unidad sobre proporcionalidad, utilizando la herramienta Idoneidad Didáctica (Daniel Esqué de los Ojos • Adriana Breda). Uniciencia is protected by Attribution-NonCommercial-NoDerivs 3.0 Unported (CC BY-NC-ND 3.0) 